\title{
DISPERSION OF MAGNETIC FIELDS IN MOLECULAR CLOUDS. II.
}

\author{
Martin Houde $^{1}$, John E. Vaillancourt ${ }^{2}$, Roger H. Hildebrand ${ }^{3,4}$, Shadi ChitsazzadeH ${ }^{1}$, And Larry Kirby ${ }^{3}$ \\ ${ }^{1}$ Department of Physics and Astronomy, The University of Western Ontario, London, ON, N6A 3K7, Canada \\ ${ }^{2}$ Division of Physics, Mathematics, \& Astronomy, California Institute of Technology, Pasadena, CA 91125, USA \\ ${ }^{3}$ Department of Astronomy and Astrophysics and Enrico Fermi Institute, The University of Chicago, Chicago, IL 60637, USA \\ ${ }^{4}$ Department of Physics, The University of Chicago, Chicago, IL 60637, USA \\ Received 2009 August 13; accepted 2009 September 30; published 2009 November 13
}

\begin{abstract}
We expand our study on the dispersion of polarization angles in molecular clouds. We show how the effect of signal integration through the thickness of the cloud as well as across the area subtended by the telescope beam inherent to dust continuum measurements can be incorporated in our analysis to correctly account for its effect on the measured angular dispersion and inferred turbulent to large-scale magnetic field strength ratio. We further show how to evaluate the turbulent magnetic field correlation scale from polarization data of sufficient spatial resolution and high enough spatial sampling rate. We apply our results to the molecular cloud OMC-1, where we find a turbulent correlation length of $\delta \approx 16 \mathrm{mpc}$, a turbulent to large-scale magnetic field strength ratio of approximately 0.5 , and a plane-of-the-sky large-scale magnetic field strength of approximately $760 \mu \mathrm{G}$.
\end{abstract}

Key words: ISM: clouds - ISM: magnetic fields - polarization - turbulence

\section{INTRODUCTION}

The observational determination of the turbulent energy content within the magnetic field is important for understanding the role of magnetic fields in the star formation process. It provides, for example, some measure of the amount of turbulent energy contained in the gas, while the further determination of the turbulent to large-scale magnetic field strength ratio can in principle be used to evaluate the large-scale magnetic strength with the so-called Chandrasekhar-Fermi equation (Chandrasekhar \& Fermi 1953).

The determination of the turbulent to large-scale magnetic field strength ratio was the subject of a recent publication (Hildebrand et al. 2009; hereafter Paper I) where it was shown how this parameter can be precisely extracted from dust continuum polarization data through a careful analysis of polarization angle differences as a function of the distance between pair of points where measurements were made (i.e., the angular dispersion function). It was described how the evaluation of the turbulent polarization angular dispersion can be achieved without assuming any model for the large-scale magnetic field component about which this dispersion is calculated. This is an important development since no model will perfectly fit the true morphology of the large-scale magnetic field. Fits to a model will therefore lead to inaccurate estimates of the angular dispersion. This error would then be propagated in the determination, for example, of the large-scale magnetic field strength when the Chandrasekhar-Fermi equation is used.

In the second paper on the subject, we generalize our analysis of Paper I by including the process of signal integration through the thickness of the cloud as well as across the area subtended by the telescope beam inherent to dust continuum measurements. It has long been recognized that the amount of angular dispersion measured in a polarization map is reduced by any integration process (Myers \& Goodman 1991), as has since been studied and demonstrated through numerical simulations (Ostriker et al. 2001; Padoan et al. 2001; Heitsch et al. 2001; Kudoh \& Basu 2003; Falceta-Gonçalves et al. 2008). The effect of integration through the thickness of a cloud has been considered by Myers \& Goodman (1991) in their studies of the optical polarization of dark clouds, while the further inclusion of integration across the area subtended by the telescope beam and ensuing consequences on measurements were investigated through simulations by Heitsch et al. (2001), Wiebe \& Watson (2004), and FalcetaGonçalves et al. (2008).

We will start with a generalization of the problem considered in Paper I by deriving the cloud- and beam-integrated dispersion function in Section 2, which will then be solved for the special case of Gaussian turbulent autocorrelation and beam profile functions. In Section 3, we apply our analysis to dust continuum polarization data obtained with SHARP (Novak et al. 2004; Li et al. 2006, 2008) for the molecular cloud OMC-1. We show how the turbulent correlation length scale for this cloud can be evaluated with the corresponding polarization data. We then use these results to determine the number of independent turbulent cells contained in the column of dust probed with our measurements and calculate the turbulent to large-scale magnetic field strength ratio corrected for the signal integration process. We provide a detailed discussion of our results in Section 4 and end with a summary in Section 5. Detailed derivations resulting in the relations and functions used in these sections, description of the data analysis, as well as a list of variables and functions will be found in the appendices at the end of the paper.

\section{ANALYSIS}

\subsection{The Cloud-and Beam-integrated Angular Dispersion} Function

In Paper I, the following equation was introduced (see their Equations (A4) and (20)) for the analytical derivation of the dispersion in polarization angles within a turbulent molecular cloud

$$
\langle\cos [\Delta \Phi(\ell)]\rangle=\frac{\langle\mathbf{B}(\mathbf{x}) \cdot \mathbf{B}(\mathbf{x}+\ell)\rangle}{\left[\left\langle B^{2}(\mathbf{x})\right\rangle\left\langle B^{2}(\mathbf{x}+\ell)\right\rangle\right]^{1 / 2}},
$$

where $\Delta \Phi(\ell) \equiv \Phi(\mathbf{x})-\Phi(\mathbf{x}+\ell)$ is the difference in the polarization angle $\Phi$ measured at two positions separated by a distance $\ell$ and $\langle\cdots\rangle$ denotes an average. As was then shown, Equation (1) (and others that derive from it) applies equally well 
to a three-dimensional magnetic field or its two-dimensional projection onto a plane; for the purpose of this paper we consider $\mathbf{B}(\mathbf{x})$ to be the plane-of-the-sky projected magnetic field, as usually probed with dust continuum polarization measurements. The distance $\ell$ is also confined to the plane-of-the-sky, unless otherwise noted. We assume that the magnetic field $\mathbf{B}(\mathbf{x})$ is composed of a large-scale, structured field, $\mathbf{B}_{0}(\mathbf{x})$, and a turbulent (or random) component, $\mathbf{B}_{\mathrm{t}}(\mathbf{x})$, such that

$$
\mathbf{B}(\mathbf{x})=\mathbf{B}_{0}(\mathbf{x})+\mathbf{B}_{\mathrm{t}}(\mathbf{x}) .
$$

We must note, however, that the magnetic field direction inferred from polarization data is actually the result of some averaging process as one integrates through the thickness of the cloud along the line of sight as well as across the area subtended by the telescope beam. We therefore first define the normalized magnetic field vector

$$
\mathbf{b}(\mathbf{r}, z) \equiv \frac{\mathbf{B}(\mathbf{r}, z)}{\left\langle B^{2}(\mathbf{r}, z)\right\rangle^{1 / 2}}
$$

and infer a mean direction for the cloud-integrated magnetic field through the following weighted integral of $\mathbf{b}(\mathbf{r}, z)$ :

$$
\overline{\mathbf{b}}(\mathbf{r}) \equiv \iint H(\mathbf{r}-\mathbf{a})\left[\frac{1}{\Delta} \int_{0}^{\Delta} F(\mathbf{a}, z) \mathbf{b}(\mathbf{a}, z) d z\right] d^{2} a .
$$

The two-dimensional (convolution) integral in Equation (4) is over all space such that for the three-dimensional position vector $\mathbf{x}, \mathbf{r}$ is the two-dimensional polar radius vector on the plane-ofthe-sky and $z$ is the depth within the cloud. That is,

$$
\mathbf{x}=r \mathbf{e}_{r}+z \mathbf{e}_{z}
$$

with $\mathbf{e}_{r}$ and $\mathbf{e}_{z}$ being the unit basis vectors along $\mathbf{r}$ and the $z-$ axis (which is oriented along the line of sight), respectively. The beam profile density is denoted by $H(\mathbf{r})$, while the weighting function $F(\mathbf{r}, z) \geqslant 0$ is the polarized emission associated with the magnetic field $\mathbf{b}(\mathbf{r}, z){ }^{5}$ Please note that even though $\mathbf{b}(\mathbf{r}, z)$ is normalized $\overline{\mathbf{b}}(\mathbf{r})$ is not, and while the normalization by $\Delta$ in Equation (4) is not essential it is included for convenience. Moreover, the quantity $\Delta$ is for the maximum depth of the cloud along any line of sight; the detailed behavior of the $F(\mathbf{r}, z)$ function thus ensures that Equation (4) is exact even when the depth of the cloud is expected to vary with position on the plane-of-the-sky.

The normalization of the magnetic field vector through the cloud is warranted because the amount of polarized emission in a given region is not a function of the strength of the magnetic field itself. Because of this we must now consider a slightly different relation for determining the dispersion function. That is, we replace Equation (1) with

$$
\langle\cos [\Delta \Phi(\ell)]\rangle \equiv \frac{\langle\overline{\mathbf{b}}(\mathbf{r}) \cdot \overline{\mathbf{b}}(\mathbf{r}+\boldsymbol{\ell})\rangle}{\left[\left\langle\bar{b}^{2}(\mathbf{r})\right\rangle\left\langle\bar{b}^{2}(\mathbf{r}+\ell)\right\rangle\right]^{1 / 2}} .
$$

In what follows, we introduce a level of idealization necessary for obtaining a quantitative measure of the turbulent component

\footnotetext{
5 The measured linear polarization orientation is normal to that of the associated plane-of-the-sky magnetic field when detecting dust continuum emission (at submillimeter wavelengths, for example), not parallel to it as could be inferred from Equation (4). This is irrelevant to our analysis, however, as we are using the polarized emission as a weighting function to define a mean orientation for the integrated magnetic field.
}

of the magnetic field in molecular clouds. We assume stationarity, homogeneity, and isotropy in the magnetic field strength, as well as statistical independence between its large-scale and turbulent components. We therefore have the following averages at points $\mathbf{x}$ and $\mathbf{y}$ :

$$
\begin{gathered}
\left\langle\mathbf{B}_{0}(\mathbf{x})\right\rangle=\mathbf{B}_{0}(\mathbf{x}), \\
\left\langle\mathbf{B}_{\mathrm{t}}(\mathbf{x})\right\rangle=0 \\
\left\langle\mathbf{B}_{0}(\mathbf{x}) \cdot \mathbf{B}_{\mathrm{t}}(\mathbf{y})\right\rangle=\left\langle\mathbf{B}_{0}(\mathbf{x})\right\rangle \cdot\left\langle\mathbf{B}_{\mathrm{t}}(\mathbf{y})\right\rangle=0
\end{gathered}
$$

and

$$
\begin{aligned}
\left\langle\mathbf{B}_{0}^{2}(\mathbf{x})\right\rangle & =\left\langle\mathbf{B}_{0}^{2}(\mathbf{y})\right\rangle=\left\langle B_{0}^{2}\right\rangle, \\
\left\langle\mathbf{B}_{\mathrm{t}}^{2}(\mathbf{x})\right\rangle & =\left\langle\mathbf{B}_{\mathrm{t}}^{2}(\mathbf{y})\right\rangle=\left\langle B_{\mathrm{t}}^{2}\right\rangle .
\end{aligned}
$$

It is straightforward to show that the homogeneity in the field strength renders the field normalization of Equation (3) inconsequential in Equation (6) and we can therefore equally write

$$
\langle\cos [\Delta \Phi(\ell)]\rangle=\frac{\langle\overline{\mathbf{B}}(\mathbf{r}) \cdot \overline{\mathbf{B}}(\mathbf{r}+\ell)\rangle}{\left[\left\langle\bar{B}^{2}(\mathbf{r})\right\rangle\left\langle\bar{B}^{2}(\mathbf{r}+\ell)\right\rangle\right]^{1 / 2}},
$$

with the cloud- and beam-integrated magnetic field

$$
\overline{\mathbf{B}}(\mathbf{r})=\iint H(\mathbf{r}-\mathbf{a})\left[\frac{1}{\Delta} \int_{0}^{\Delta} F(\mathbf{a}, z) \mathbf{B}(\mathbf{a}, z) d z\right] d^{2} a
$$

and where the assumed isotropy in the distance $(\ell=|\ell|)$ was incorporated. Equation (9) is the relation we will use to estimate the angular dispersion function.

\subsection{The Integrated Magnetic Field Autocorrelation Function}

In view of the assumed stationarity and isotropy the integrated magnetic field autocorrelation function $\langle\overline{\mathbf{B}} \cdot \overline{\mathbf{B}}(\ell)\rangle \equiv$ $\langle\overline{\mathbf{B}}(\mathbf{r}) \cdot \overline{\mathbf{B}}(\mathbf{r}+\ell)\rangle$ can be expressed as (see Equations (A1)-(A5))

$$
\begin{aligned}
\langle\overline{\mathbf{B}} \cdot \overline{\mathbf{B}}(\ell)\rangle= & \iiint \int H(\mathbf{a}) H\left(\mathbf{a}^{\prime}+\ell\right) \\
& \times\left[\frac{2}{\Delta} \int_{0}^{\Delta}\left(1-\frac{u}{\Delta}\right) R_{F}(v, u) R_{\mathbf{B}}(v, u) d u\right] d^{2} a^{\prime} d^{2} a,
\end{aligned}
$$

where we introduced the (assumed statistically independent) autocorrelation functions for the (non-integrated) magnetic field and the polarized emission

$$
\begin{gathered}
R_{\mathbf{B}}(v, u)=\left\langle\mathbf{B}(\mathbf{a}, z) \cdot \mathbf{B}\left(\mathbf{a}^{\prime}, z^{\prime}\right)\right\rangle \\
R_{F}(v, u)=\left\langle F(\mathbf{a}, z) F\left(\mathbf{a}^{\prime}, z^{\prime}\right)\right\rangle
\end{gathered}
$$

with $u=\left|z^{\prime}-z\right|$ and $v=\left|\mathbf{a}^{\prime}-\mathbf{a}\right|$. These autocorrelations can be further broken down through the decomposition of the magnetic field and the polarized emission into their respective large-scale and turbulent components (see Equation (2))

$$
\begin{gathered}
\mathbf{B}(\mathbf{a}, z)=\mathbf{B}_{0}(\mathbf{a}, z)+\mathbf{B}_{\mathrm{t}}(\mathbf{a}, z), \\
F(\mathbf{a}, z)=F_{0}(\mathbf{a}, z)+F_{\mathrm{t}}(\mathbf{a}, z) .
\end{gathered}
$$

Upon assuming the same statistical properties for the components of $F(\mathbf{a}, z)$ as those expressed in Equations (7) for $\mathbf{B}(\mathbf{r}, z)$, Equations (12) and (13) transform to

$$
\begin{aligned}
& R_{\mathbf{B}}(v, u)=R_{\mathbf{B}, 0}(v, u)+R_{\mathbf{B}, \mathrm{t}}(v, u), \\
& R_{F}(v, u)=R_{F, 0}(v, u)+R_{F, \mathrm{t}}(v, u)
\end{aligned}
$$


with

$$
\begin{gathered}
R_{\mathbf{B}, j}(v, u)=\left\langle\mathbf{B}_{j}(\mathbf{a}, z) \cdot \mathbf{B}_{j}\left(\mathbf{a}^{\prime}, z^{\prime}\right)\right\rangle \\
R_{F, j}(v, u)=\left\langle F_{j}(\mathbf{a}, z) F_{j}\left(\mathbf{a}^{\prime}, z^{\prime}\right)\right\rangle
\end{gathered}
$$

where $j=$ ' 0 ' or ' $t$ ' for the large-scale and turbulent components, respectively.

The solution to our problem is reduced to solving Equation (11) given the different autocorrelation functions; the equation to be used for determining the dispersion function then becomes

$$
\langle\cos [\Delta \Phi(\ell)]\rangle=\frac{\langle\overline{\mathbf{B}} \cdot \overline{\mathbf{B}}(\ell)\rangle}{\langle\overline{\mathbf{B}} \cdot \overline{\mathbf{B}}(0)\rangle} .
$$

\subsection{Solution Using Gaussian Turbulent Autocorrelation and Beam Profile Functions}

In order to solve Equation (20) we must specify, at least to some level, the characteristics of the different autocorrelation functions, as well as the telescope beam profile. We therefore define the following

$$
\begin{gathered}
R_{\mathbf{B}}(v, u)=R_{\mathbf{B}, 0}(v, u)+\left\langle B_{\mathrm{t}}^{2}\right\rangle e^{-\left(v^{2}+u^{2}\right) / 2 \delta^{2}} \\
R_{F}(v, u)=R_{F, 0}(v, u)+\left\langle F_{\mathrm{t}}^{2}\right\rangle e^{-\left(v^{2}+u^{2}\right) / 2 \delta^{2}},
\end{gathered}
$$

where the magnetic field and polarized emission autocorrelations each have large-scale and turbulent (second term on the right-hand sides) components. The correlation length scales for the turbulent magnetic field and polarized emission are $\delta$ and $\delta^{\prime}$, respectively, and, as is implied through Equations (21) and (22), we assume the turbulence to be isotropic. Furthermore, $\delta$ and $\delta^{\prime}$ are taken to be much smaller than the thickness of the cloud (i.e., $\delta \ll \Delta$ and $\delta^{\prime} \ll \Delta$ ). The beam profile is defined with

$$
H(\mathbf{r})=\frac{1}{2 \pi W^{2}} e^{-r^{2} / 2 W^{2}},
$$

where $W$ is the beam "radius."

Under these constraints and definitions Equation (11) can be analytically solved to yield (see Equations (A6)-(A8))

$$
\begin{aligned}
\langle\overline{\mathbf{B}} \cdot \overline{\mathbf{B}}(\ell)\rangle \simeq & \left\langle B_{0}^{2}\right\rangle\left\langle F_{0}^{2}\right\rangle\left(\langle\alpha(\ell)\rangle+\sqrt{2 \pi} \frac{\left\langle B_{\mathrm{t}}^{2}\right\rangle}{\left\langle B_{0}^{2}\right\rangle}\left\{\left[\frac{\delta^{3}}{\left(\delta^{2}+2 W^{2}\right) \Delta}\right] e^{-\ell^{2} / 2\left(\delta^{2}+2 W^{2}\right)}\right.\right. \\
& \left.+\frac{\left\langle F_{\mathrm{t}}^{2}\right\rangle}{\left\langle F_{0}^{2}\right\rangle}\left[\frac{\delta^{\prime \prime 3}}{\left(\delta^{\prime 2}+2 W^{2}\right) \Delta}\right] e^{-\ell^{2} / 2\left(\delta^{\prime 2}+2 W^{2}\right)}\right\} \\
+ & \left.\sqrt{2 \pi} \frac{\left\langle F_{\mathrm{t}}^{2}\right\rangle}{\left\langle F_{0}^{2}\right\rangle}\left[\frac{\delta^{\prime 3}}{\left(\delta^{\prime 2}+2 W^{2}\right) \Delta}\right] e^{-\ell^{2} / 2\left(\delta^{\prime 2}+2 W^{2}\right)}\right)
\end{aligned}
$$

with

$$
\begin{aligned}
\left\langle B_{0}^{2}\right\rangle & =R_{\mathbf{B}, 0}(0,0) \\
\left\langle F_{0}^{2}\right\rangle & =R_{F, 0}(0,0) \\
\delta^{\prime \prime} & =\frac{\delta \delta^{\prime}}{\sqrt{\delta^{2}+\delta^{\prime 2}}}
\end{aligned}
$$

and the normalized large-scale function averaging of the turbulent components through the column of dust probed by the corresponding polarization measurements. Indeed, it is apparent that each of the turbulent contributions in Equation (24) scales with a term such as

$$
N^{-1}=\frac{\sqrt{2 \pi} \delta^{3}}{\left(\delta^{2}+2 W^{2}\right) \Delta},
$$

where $N$ is nothing more than the number of independent turbulent cells (for the magnetic field in this case) contained in the column of dust probed observationally, as could have been intuitively guessed. We also note that for cases where the telescope beam radius were to be much smaller than the size of a turbulent cell (e.g., $\delta \gg W$ ) we recover

$$
N=\frac{\Delta}{\sqrt{2 \pi} \delta} .
$$

The number of independent turbulent cells would thus be accounted for by those cells that lie along the line of sight through the thickness of the cloud at a given point on its surface, as would also be expected intuitively (Myers \& Goodman 1991).

Inserting Equations (24)-(28) into Equation (20) we can write

$$
\begin{aligned}
1 & -\langle\cos [\Delta \Phi(\ell)]\rangle=\frac{\left\langle B_{0}^{2}\right\rangle\left\langle F_{0}^{2}\right\rangle}{\langle\overline{\mathbf{B}} \cdot \overline{\mathbf{B}}(0)\rangle}([\langle\alpha(0)\rangle-\langle\alpha(\ell)\rangle] \\
& +\sqrt{2 \pi} \frac{\left\langle B_{\mathrm{t}}^{2}\right\rangle}{\left\langle B_{0}^{2}\right\rangle}\left\{\left[\frac{\delta^{3}}{\left(\delta^{2}+2 W^{2}\right) \Delta}\right]\left[1-e^{-\ell^{2} / 2\left(\delta^{2}+2 W^{2}\right)}\right]\right. \\
& \left.+\frac{\left\langle F_{\mathrm{t}}^{2}\right\rangle}{\left\langle F_{0}^{2}\right\rangle}\left[\frac{\delta^{\prime \prime 3}}{\left(\delta^{\prime \prime 2}+2 W^{2}\right) \Delta}\right]\left[1-e^{-\ell^{2} / 2\left(\delta^{\prime \prime 2}+2 W^{2}\right)}\right]\right\} \\
& \left.+\sqrt{2 \pi} \frac{\left\langle F_{\mathrm{t}}^{2}\right\rangle}{\left\langle F_{0}^{2}\right\rangle}\left[\frac{\delta^{\prime 3}}{\left(\delta^{\prime 2}+2 W^{2}\right) \Delta}\right]\left[1-e^{-\ell^{2} / 2\left(\delta^{\prime 2}+2 W^{2}\right)}\right]\right) .
\end{aligned}
$$

The first term within parentheses on the right-hand side is, as was previously stated, due to the large-scale structure in the magnetic field and the polarized emission; it does not involve turbulence. We can expand this term using a Taylor series with

$$
\langle\alpha(0)\rangle-\langle\alpha(\ell)\rangle=\sum_{j=1}^{\infty} a_{2 j} \ell^{2 j},
$$

where the summation is performed only on even values for $2 j$, since $\langle\alpha(\ell)\rangle$ is isotropic in $\ell$ (see Section A.2).

Although the remaining terms in Equation (31) are all due to turbulence, their respective contributions have, in part, different origins. More importantly, it should be clear that the total turbulent component is not only due to turbulence in the magnetic field but can also arise from the presence of turbulence in the polarized emission. For example, the third term within parentheses on the right-hand side of Equation (31) can be interpreted as a contribution due to random changes in polarized

$$
\langle\alpha(\ell)\rangle=\iiint \int H(\mathbf{a}) H\left(\mathbf{a}^{\prime}+\ell\right)\left\{\frac{2}{\Delta} \int_{0}^{\Delta}\left(1-\frac{u}{\Delta}\right)\left[\frac{R_{F, 0}(v, u)}{\left\langle F_{0}^{2}\right\rangle}\right]\left[\frac{R_{\mathbf{B}, 0}(v, u)}{\left\langle B_{0}^{2}\right\rangle}\right] d u\right\} d^{2} a^{\prime} d^{2} a
$$

It is expected that this large-scale function will usually dominate the other terms in Equation (24), since these all result from the emission at different positions in the cloud where the magnetic field also changes orientation. It follows that the measured (i.e., 
integrated) orientation associated with the polarized emission, and therefore the deduced magnetic field orientation (i.e., the polarization angle), will also accordingly fluctuate randomly.

The last term in Equation (31) seems to imply that the turbulent component in polarized emission will contribute to the turbulent angular dispersion even in cases where the magnetic field is uniform (and does not have a turbulent component). But this is an artifact of the way we analytically evaluate the dispersion function through Equation (31), which does not perfectly mimic the way polarization measurements are accomplished (see Section 4). In reality, polarization measurements are made on a point-by-point basis, and the dispersion function is calculated through an average of (the cosine of) angle differences as a function of the displacement $\ell$ (see Equation (1) in Paper I) not through the evaluation of an autocorrelation function such as given in Equation (11). It follows that a perfectly uniform magnetic field could never lead to a measurable angular dispersion; we will therefore not include in our analysis the corresponding contribution in Equation (31).

Now consider the following

$$
\begin{aligned}
\frac{\langle\overline{\mathbf{B}} \cdot \overline{\mathbf{B}}(0)\rangle}{\left\langle B_{0}^{2}\right\rangle\left\langle F_{0}^{2}\right\rangle} \simeq & \langle\alpha(0)\rangle \\
\simeq & \iiint \int H(\mathbf{a}) H\left(\mathbf{a}^{\prime}\right)\left\{\frac{2}{\Delta} \int_{0}^{\Delta}\left(1-\frac{u}{\Delta}\right)\right. \\
& \left.\times\left[\frac{R_{F, 0}(v, u)}{\left\langle F_{0}^{2}\right\rangle}\right]\left[\frac{R_{\mathbf{B}, 0}(v, u)}{\left\langle B_{0}^{2}\right\rangle}\right] d u\right\} d^{2} a^{\prime} d^{2} a,
\end{aligned}
$$

where we have taken advantage of the fact that we expect that the large-scale component $\langle\alpha(0)\rangle$ dominates the turbulent terms in Equation (24) (when $\ell=0$ ), as was previously stated. Because the autocorrelations present in the integrand of the onedimensional integral are normalized it follows that this integral, which we denote as $A\left(\left|\mathbf{a}^{\prime}-\mathbf{a}\right|\right) / 2$, will always be less than $\Delta / 2$ and thus

$$
\begin{aligned}
\frac{\langle\overline{\mathbf{B}} \cdot \overline{\mathbf{B}}(0)\rangle}{\left\langle B_{0}^{2}\right\rangle\left\langle F_{0}^{2}\right\rangle} & \simeq \frac{1}{\Delta} \iiint \int H(\mathbf{a}) H\left(\mathbf{a}^{\prime}\right) A\left(\left|\mathbf{a}^{\prime}-\mathbf{a}\right|\right) d^{2} a^{\prime} d^{2} a \\
& \equiv \frac{\Delta^{\prime}}{\Delta} \leqslant 1
\end{aligned}
$$

where the equality to unity only holds when the large-scale magnetic field and polarized emission are both uniform. The quantity $\Delta^{\prime}$ can be interpreted as the effective thickness of the cloud; this will be discussed in more details in Section 3.2.

Taking these considerations into account, Equation (31) becomes

$$
\begin{aligned}
1 & -\langle\cos [\Delta \Phi(\ell)]\rangle \simeq \sqrt{2 \pi} \frac{\left\langle B_{\mathrm{t}}^{2}\right\rangle}{\left\langle B_{0}^{2}\right\rangle}\left\{\left[\frac{\delta^{3}}{\left(\delta^{2}+2 W^{2}\right) \Delta^{\prime}}\right]\left[1-e^{-\ell^{2} / 2\left(\delta^{2}+2 W^{2}\right)}\right]\right. \\
& \left.+\frac{\left\langle F_{\mathrm{t}}^{2}\right\rangle}{\left\langle F_{0}^{2}\right\rangle}\left[\frac{\delta^{\prime \prime 3}}{\left(\delta^{\prime \prime 2}+2 W^{2}\right) \Delta^{\prime}}\right]\left[1-e^{-\ell^{2} / 2\left(\delta^{\prime \prime 2}+2 W^{2}\right)}\right]\right\}+\sum_{j=1}^{\infty} a_{2 j}^{\prime} \ell^{2 j},
\end{aligned}
$$

with $a_{2 j}^{\prime}=\left(\Delta / \Delta^{\prime}\right) a_{2 j}$. If we limit ourselves to small enough displacements such that $\ell$ is less than a few times the beam radius $W$, then the large-scale term becomes small enough to be adequately described by the first term in the Taylor expansion of Equation (32) and Equation (35) can be approximated to

$$
\begin{aligned}
1 & -\langle\cos [\Delta \Phi(\ell)]\rangle \simeq \sqrt{2 \pi} \frac{\left\langle B_{\mathrm{t}}^{2}\right\rangle}{\left\langle B_{0}^{2}\right\rangle}\left\{\left[\frac{\delta^{3}}{\left(\delta^{2}+2 W^{2}\right) \Delta^{\prime}}\right]\left[1-e^{-\ell^{2} / 2\left(\delta^{2}+2 W^{2}\right)}\right]\right. \\
+ & \left.\frac{\left\langle F_{\mathrm{t}}^{2}\right\rangle}{\left\langle F_{0}^{2}\right\rangle}\left[\frac{\delta^{\prime \prime 3}}{\left(\delta^{\prime \prime 2}+2 W^{2}\right) \Delta^{\prime}}\right]\left[1-e^{-\ell^{2} / 2\left(\delta^{\prime \prime 2}+2 W^{2}\right)}\right]\right\}+a_{2}^{\prime} \ell^{2}
\end{aligned}
$$

or alternatively

$$
\begin{gathered}
\left\langle\Delta \Phi^{2}(\ell)\right\rangle \simeq 2 \sqrt{2 \pi} \frac{\left\langle B_{\mathrm{t}}^{2}\right\rangle}{\left\langle B_{0}^{2}\right\rangle}\left\{\left[\frac{\delta^{3}}{\left(\delta^{2}+2 W^{2}\right) \Delta^{\prime}}\right]\left[1-e^{-\ell^{2} / 2\left(\delta^{2}+2 W^{2}\right)}\right]\right. \\
\left.+\frac{\left\langle F_{\mathrm{t}}^{2}\right\rangle}{\left\langle F_{0}^{2}\right\rangle}\left[\frac{\delta^{\prime \prime 3}}{\left(\delta^{\prime \prime 2}+2 W^{2}\right) \Delta^{\prime}}\right]\left[1-e^{-\ell^{2} / 2\left(\delta^{\prime \prime 2}+2 W^{2}\right)}\right]\right\}+m^{2} \ell^{2},
\end{gathered}
$$

where $m^{2}=2 a_{2}^{\prime}$.

\section{RESULTS}

\subsection{The Polarized Emission}

Ideally one would intend to use Equation (36) for the dispersion function (or alternatively Equation (37)) to determine the ratio of the (square of the) turbulent to large-scale magnetic field strength $\left\langle B_{\mathrm{t}}^{2}\right\rangle /\left\langle B_{0}^{2}\right\rangle$. As was shown in Paper I, this dispersion function is readily evaluated from polarization data and the aforementioned ratio could be used to calculate, for example, the large-scale magnetic field strength $\left\langle B_{0}^{2}\right\rangle^{1 / 2}$ through the Chandrasekhar-Fermi equation (Chandrasekhar \& Fermi 1953). It should be clear, however, that in order to precisely achieve such a goal we must find a way to determine other parameters such as the turbulent correlation scales $\delta$ and $\delta^{\prime}$, the effective cloud thickness $\Delta^{\prime}$, and the ratio of the turbulent to large-scale polarized emission $\left\langle F_{\mathrm{t}}^{2}\right\rangle /\left\langle F_{0}^{2}\right\rangle$.

The polarized emission, in particular, is problematic. This is because we do not have direct information on the weighting function $F(\mathbf{r}, z)$ or its integrated counterpart

$$
\bar{F}(\mathbf{r})=\iint H(\mathbf{r}-\mathbf{a})\left[\frac{1}{\Delta} \int_{0}^{\Delta} F(\mathbf{a}, z) d z\right] d^{2} a .
$$

Although the integrated polarized flux $\bar{P}(\mathbf{r})$ is contained in polarization data sets obtained with dust continuum polarimeters such as Hertz (Dotson et al. 2009), SCUBA (Matthews et al. 2009), and SHARP (Vaillancourt et al. 2008), it does not correspond to the quantity defined in Equation (38). Instead the polarized flux is observationally determined through the measurement of the integrated Stokes parameters

$$
\begin{aligned}
& \bar{Q}(\mathbf{r})=\iint H(\mathbf{r}-\mathbf{a})\left[\frac{1}{\Delta} \int_{0}^{\Delta} Q(\mathbf{a}, z) d z\right] d^{2} a \\
& \bar{U}(\mathbf{r})=\iint H(\mathbf{r}-\mathbf{a})\left[\frac{1}{\Delta} \int_{0}^{\Delta} U(\mathbf{a}, z) d z\right] d^{2} a
\end{aligned}
$$

with

$$
\bar{P}(\mathbf{r})=\sqrt{\bar{Q}^{2}(\mathbf{r})+\bar{U}^{2}(\mathbf{r})} .
$$

Although these parameters will also exhibit large-scale and turbulent components, they do not provide us with any means for disentangling the turbulent contributions $\left\langle B_{\mathrm{t}}^{2}\right\rangle$ and $\left\langle F_{\mathrm{t}}^{2}\right\rangle$ due to the magnetic field and the polarized emission they contain. 
We must then resort to some approximation(s) if we are to make any progress.

Perhaps the most obvious difference between the two turbulent terms on the right-hand side of Equation (35) is that the first one is of first order in the square of a turbulent to large-scale strength ratio while the other is of second order. If we assume that such a ratio is a fraction of unity, then the second-order term can be neglected. This amounts to neglecting the contribution of the turbulent polarized emission in Equation (35); this is the line of attack we will use from now on. We therefore write that

$$
\begin{aligned}
1-\langle\cos [\Delta \Phi(\ell)]\rangle \simeq & \sqrt{2 \pi} \frac{\left\langle B_{\mathrm{t}}^{2}\right\rangle}{\left\langle B_{0}^{2}\right\rangle}\left[\frac{\delta^{3}}{\left(\delta^{2}+2 W^{2}\right) \Delta^{\prime}}\right] \\
& \times\left[1-e^{-\ell^{2} / 2\left(\delta^{2}+2 W^{2}\right)}\right]+\sum_{j=1}^{\infty} a_{2 j}^{\prime} \ell^{2 j},
\end{aligned}
$$

while for displacements $\ell$ less than a few times $W$ we keep only the first $\ell^{2}$ term in the Taylor expansion

$$
\begin{aligned}
1-\langle\cos [\Delta \Phi(\ell)]\rangle \simeq & \sqrt{2 \pi} \frac{\left\langle B_{\mathrm{t}}^{2}\right\rangle}{\left\langle B_{0}^{2}\right\rangle}\left[\frac{\delta^{3}}{\left(\delta^{2}+2 W^{2}\right) \Delta^{\prime}}\right] \\
& \times\left[1-e^{-\ell^{2} / 2\left(\delta^{2}+2 W^{2}\right)}\right]+a_{2}^{\prime} \ell^{2},
\end{aligned}
$$

or

$$
\begin{aligned}
\left\langle\Delta \Phi^{2}(\ell)\right\rangle \simeq & 2 \sqrt{2 \pi} \frac{\left\langle B_{\mathrm{t}}^{2}\right\rangle}{\left\langle B_{0}^{2}\right\rangle}\left[\frac{\delta^{3}}{\left(\delta^{2}+2 W^{2}\right) \Delta^{\prime}}\right] \\
& \times\left[1-e^{-\ell^{2} / 2\left(\delta^{2}+2 W^{2}\right)}\right]+m^{2} \ell^{2} .
\end{aligned}
$$

Whether it is appropriate or not to neglect the turbulent polarized emission is an open question, which could perhaps be adequately investigated through simulations.

\subsection{The Effective Cloud Thickness}

We previously defined the effective cloud thickness $\Delta^{\prime}$ through the following relation:

$$
\begin{aligned}
\langle\alpha(0)\rangle= & \iiint \int H(\mathbf{a}) H\left(\mathbf{a}^{\prime}\right)\left\{\frac{2}{\Delta} \int_{0}^{\Delta}\left(1-\frac{u}{\Delta}\right)\right. \\
& \left.\times\left[\frac{R_{F, 0}(v, u)}{\left\langle F_{0}^{2}\right\rangle}\right]\left[\frac{R_{\mathbf{B}, 0}(v, u)}{\left\langle B_{0}^{2}\right\rangle}\right] d u\right\} d^{2} a^{\prime} d^{2} a \\
\equiv & \frac{\Delta^{\prime}}{\Delta} \leqslant 1 .
\end{aligned}
$$

We can get a sense of the nature of $\Delta^{\prime}$ by expressing $\langle\alpha(0)\rangle$ with the Fourier transform of $\langle\alpha(\ell)\rangle$ at $\ell=0$ (see Section A.2 for more details)

$$
\begin{aligned}
\langle\alpha(0)\rangle= & \frac{1}{(2 \pi)^{2}} \iint\left\|H\left(\mathbf{k}_{v}\right)\right\|^{2}\left\{\frac{1}{2 \pi} \int\left[\frac{\mathcal{R}_{0}\left(\mathbf{k}_{v}, k_{u}\right)}{\left\langle F_{0}^{2}\right\rangle\left\langle B_{0}^{2}\right\rangle}\right]\right. \\
& \left.\times \operatorname{sinc}^{2}\left(\frac{k_{u} \Delta}{2}\right) d k_{u}\right\} d^{2} k_{v},
\end{aligned}
$$

where $\mathcal{R}_{0}\left(\mathbf{k}_{v}, k_{u}\right)$ is the Fourier transform of $\mathcal{R}_{0}(v, u)=$ $R_{F, 0}(v, u) R_{\mathbf{B}, 0}(v, u)$ and $\operatorname{sinc}(x) \equiv \sin (x) / x$. It should be apparent that Equation (46) includes several effects that set the value of $\Delta^{\prime}$. For the idealized case where the autocorrelation $\mathcal{R}_{0}(v, u)$ is uniform across the cloud, its Fourier transform $\mathcal{R}_{0}\left(\mathbf{k}_{v}, k_{u}\right)$ is proportional to a Dirac distribution (more precisely, $\left.\mathcal{R}_{0}\left(\mathbf{k}_{v}, k_{u}\right)=(2 \pi)^{3}\left\langle F_{0}^{2}\right\rangle\left\langle B_{0}^{2}\right\rangle \delta\left(\mathbf{k}_{v}, k_{u}\right)\right)$ and $\Delta^{\prime}=\Delta$, as was previously stated. For more realistic cases, however, $\mathcal{R}_{0}\left(\mathbf{k}_{v}, k_{u}\right)$ will have a finite width along $k_{u}$ and $\mathbf{k}_{v}$. As is also discussed in Section A.2, the (square of) beam profile $\left\|H\left(\mathbf{k}_{v}\right)\right\|^{2}$ will filter $\mathcal{R}_{0}\left(\mathbf{k}_{v}, k_{u}\right)$ along $\mathbf{k}_{v}$ and therefore reduce the value of $\langle\alpha(0)\rangle$ (i.e., $\Delta^{\prime}<\Delta$ ) through the exclusion of spectral modes located outside the bandwidth subtended by $\left\|H\left(\mathbf{k}_{v}\right)\right\|^{2}$. A more important filtering effect due to the finite spectral width of $\mathcal{R}_{0}\left(\mathbf{k}_{v}, k_{u}\right)$ along $k_{u}$ is expected, however, as it is severely truncated by the integration process through the cloud thickness $\Delta$. This is clearly assessed by the presence $\operatorname{sinc}^{2}\left(k_{u} \Delta / 2\right)$ in Equation (46) and the subsequent integration on $k_{u}$. Because of the assumed large difference between the cloud thickness and the beam radius (i.e., $W \ll \Delta$ ) we expect that this effect will dominate in determining the value of $\langle\alpha(0)\rangle$.

If we now introduce $\Delta k_{u}$ the spectral width of $\mathcal{R}_{0}\left(\mathbf{k}_{v}, k_{u}\right)$ along $k_{u}$ with the following definition:

$$
2 \Delta k_{u} \mathcal{R}_{0}\left(\mathbf{k}_{v}, 0\right) \equiv \int \mathcal{R}_{0}\left(\mathbf{k}_{v}, k_{u}\right) d k_{u},
$$

then we can further approximate ( since $\Delta k_{u} \gg \Delta^{-1}$ )

$$
\begin{aligned}
\int \mathcal{R}_{0}\left(\mathbf{k}_{v}, k_{u}\right) \operatorname{sinc}^{2}\left(\frac{k_{u} \Delta}{2}\right) d k_{u} & \sim \mathcal{R}_{0}\left(\mathbf{k}_{v}, 0\right) \int \operatorname{sinc}^{2}\left(\frac{k_{u} \Delta}{2}\right) d k_{u} \\
& \sim \frac{2 \pi}{\Delta} \mathcal{R}_{0}\left(\mathbf{k}_{v}, 0\right) \\
& \sim \frac{2 \pi}{\Delta}\left[\frac{1}{2 \Delta k_{u}} \int \mathcal{R}_{0}\left(\mathbf{k}_{v}, k_{u}\right) d k_{u}\right] .
\end{aligned}
$$

Inserting this last relation into Equation (46), with the assumption that the filtering due to the telescope beam is negligible compared to that due to integration through the thickness of the cloud, yields

$$
\begin{aligned}
\langle\alpha(0)\rangle & \sim\left(\frac{2 \pi}{\Delta}\right)\left(\frac{1}{2 \Delta k_{u}}\right)\left\{\frac{1}{(2 \pi)^{3}} \iiint\left[\frac{\mathcal{R}_{0}\left(\mathbf{k}_{v}, k_{u}\right)}{\left\langle F_{0}^{2}\right\rangle\left\langle B_{0}^{2}\right\rangle}\right] d^{3} k\right\} \\
& \sim\left(\frac{\pi}{\Delta}\right)\left(\frac{1}{\Delta k_{u}}\right) \\
& \equiv \frac{\Delta^{\prime}}{\Delta}
\end{aligned}
$$

We then find

$$
\Delta k_{u} \sim \frac{\pi}{\Delta^{\prime}} .
$$

Because of the way the Fourier transform relates the width of $\mathcal{R}_{0}\left(\mathbf{k}_{v}, k_{u}\right)$ to that of $\mathcal{R}_{0}(v, u), \Delta^{\prime}$ can therefore be advantageously interpreted as the width of the large-scale autocorrelation function $\mathcal{R}_{0}(v, u)$ (if we assume isotropy, then the width is the same along $u$ or $v$ ).

Unfortunately, we do not have access to $\mathcal{R}_{0}(v, u)$ when mapping the polarization of dust emission in a molecular cloud. However, we can probably get a decent approximation for $\Delta^{\prime}$ through the shape of the autocorrelation function of the aforementioned cloud- and beam-integrated polarized flux $\bar{P}(\mathbf{r})$. This function is defined with

$$
\left\langle\bar{P}^{2}(\ell)\right\rangle \equiv\langle\bar{P}(\mathbf{r}) \bar{P}(\mathbf{r}+\ell)\rangle
$$




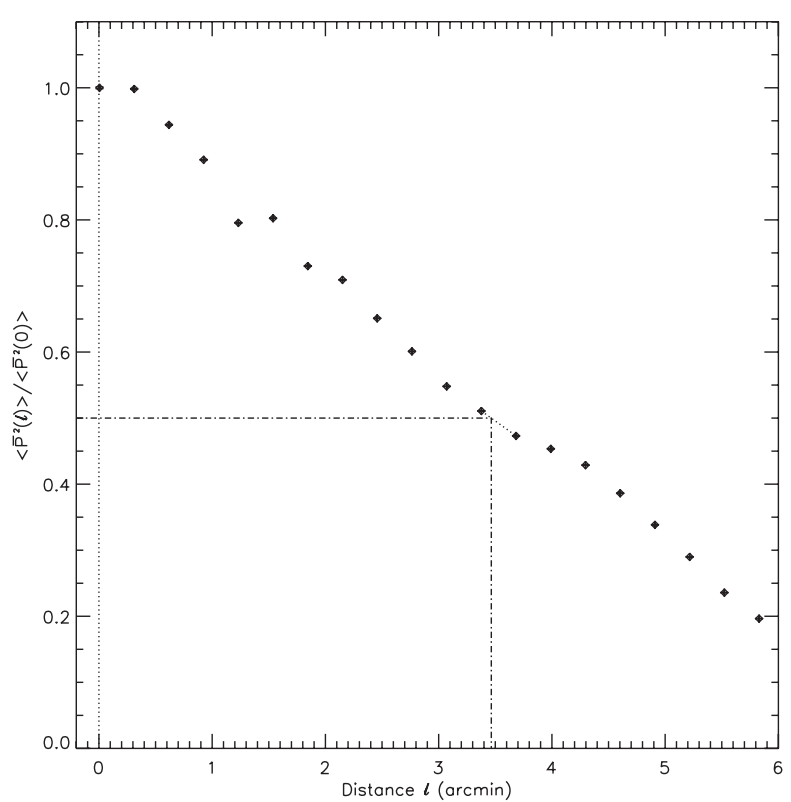

Figure 1. Normalized autocorrelation function of the integrated polarized flux as calculated using the previously published $350 \mu \mathrm{m}$ Hertz data for OMC-1 (Houde et al. 2004; Hildebrand et al. 2009). We chose the width at half magnitude to determine the value for the effective depth of OMC-1; we therefore have $\Delta^{\prime} \approx 3.5$.

Albeit this is an integrated quantity, it does provide a good sense of the proportion of the cloud that contains the bulk of the polarized flux, which is used as the weighting function for the magnetic field in our analysis (see Equation (10)). We also note that this autocorrelation is a function of the distance $\ell$ on the surface of the cloud, not through its depth. But this is consistent with the isotropy assumption from which it is expected that a molecular cloud will have similar characteristics through its depth and across its surface. It therefore seems reasonable to associate the width of $\left\langle\bar{P}^{2}(\ell)\right\rangle$ with the effective depth of the cloud.

For the case of OMC-1 we use previously published Hertz data $^{6}$ (Houde et al. 2004; Hildebrand et al. 2009) to evaluate the effective depth of the cloud. The result is presented in Figure 1, where the normalized autocorrelation function of the integrated normalized flux is shown. As indicated on the graph, we have arbitrarily chosen the width at half magnitude as the value for $\Delta^{\prime}$; we will therefore set $\Delta^{\prime} \approx 3.5$ for OMC-1 for the calculations that will follow. Also notably, we redefine $N$ as follows (see Equation (29)):

$$
N=\frac{\left(\delta^{2}+2 W^{2}\right) \Delta^{\prime}}{\sqrt{2 \pi} \delta^{3}}
$$

\subsection{The Turbulent Correlation Length Scale, the Turbulent to Large-scale Magnetic Field Strength Ratio, and the Large-scale Magnetic Field Strength}

Having estimated the effective depth of the cloud $\Delta^{\prime}$ from the autocorrelation function of the integrated normalized flux, we are now in a position to determine two fundamental parameters that characterize magnetic fields and turbulence in star-forming regions: the turbulent correlation length $\delta$ and the (square of the)

\footnotetext{
6 We use data obtained with Hertz instead of SHARP as the former cover a larger spatial extent and allow us to determine $\left\langle\bar{P}^{2}(\ell)\right\rangle$ over a large enough distance.
}
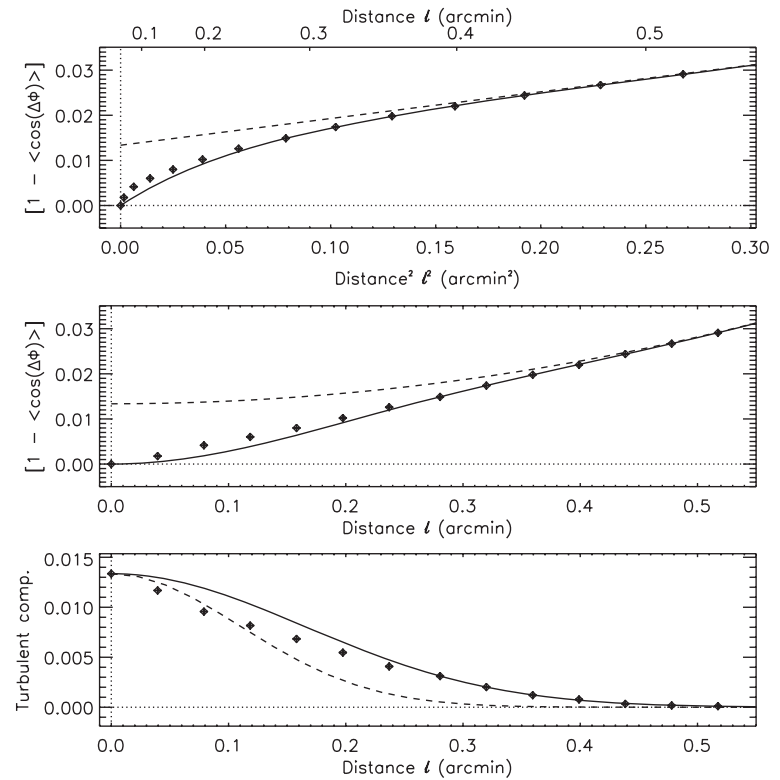

Figure 2. Dispersion function $1-\langle\cos [\Delta \Phi(\ell)]\rangle$ for OMC-1 using the $350 \mu \mathrm{m}$ data obtained with SHARP. Top: fit of Equation (43) (solid curve) to the data (symbols) when plotted as a function of $\ell^{2}$, the broken curve does not contain the correlated part of the function (see the text); middle: same as top but plotted as a function of $\ell$; bottom: the turbulent component of the dispersion function (symbols), as obtained by subtracting the data points to the broken curve in the middle graph, while the broken and solid curves are, respectively, the contribution of the (assumed Gaussian) telescope beam alone (i.e., when $\delta=0$ ) and the fit to the data (i.e., with $\delta=7^{\prime \prime} .3$ ).

turbulent to large-scale magnetic field strength ratio $\left\langle B_{\mathrm{t}}^{2}\right\rangle /\left\langle B_{0}^{2}\right\rangle$. To do so we refer to Equation (43), which we write again here for convenience

$$
\begin{aligned}
1-\langle\cos [\Delta \Phi(\ell)]\rangle \simeq & \sqrt{2 \pi} \frac{\left\langle B_{\mathrm{t}}^{2}\right\rangle}{\left\langle B_{0}^{2}\right\rangle}\left[\frac{\delta^{3}}{\left(\delta^{2}+2 W^{2}\right) \Delta^{\prime}}\right] \\
& \times\left[1-e^{-\ell^{2} / 2\left(\delta^{2}+2 W^{2}\right)}\right]+a_{2}^{\prime} \ell^{2},
\end{aligned}
$$

which is valid when the displacement $\ell$ is less than a few times $W$.

Our plan consists of using the previously published $350-\mu \mathrm{m}$ SHARP polarization map of OMC-1 (Vaillancourt et al. 2008) to evaluate the left-hand side of Equation (53) and fit our solution to the problem (i.e., the right-hand side) to the data. There are only three quantities to be simultaneously fitted for $\delta,\left\langle B_{\mathrm{t}}^{2}\right\rangle /\left\langle B_{0}^{2}\right\rangle$, and $a_{2}^{\prime}$, the first two being the parameters we are most interested in at this time. More details concerning our data analysis will be found in Appendix B.

We show in Figure 2 the result of our nonlinear fit to the aforementioned data. In the figure, the top graph shows the fit of Equation (53) (solid curve) to the data (symbols) when plotted as a function of $\ell^{2}$. The broken curve does not contain the correlated part of the function, i.e., the function

$$
\sqrt{2 \pi} \frac{\left\langle B_{\mathrm{t}}^{2}\right\rangle}{\left\langle B_{0}^{2}\right\rangle}\left[\frac{\delta^{3}}{\left(\delta^{2}+2 W^{2}\right) \Delta^{\prime}}\right]+a_{2}^{\prime} \ell^{2}
$$

is displayed to better visualize the integrated turbulent contribution (i.e., the first term) to the dispersion in relation to the large-scale component (i.e., $a_{2}^{\prime} \ell^{2}$ ) when $\ell$ is less than a few times $W$. The middle graph of Figure 2 displays the same information as the top graph but plotted as a function of $\ell$. In the bottom graph, we show the correlated turbulent component of the dispersion function (symbols) obtained by subtracting the 
data to the broken curve in the middle graph, which in our model (solid curve) corresponds to

$$
\begin{aligned}
b^{2}(\ell) & \equiv \frac{\left\langle\overline{\mathbf{B}}_{\mathrm{t}} \cdot \overline{\mathbf{B}}_{\mathrm{t}}(\ell)\right\rangle}{\left\langle B_{0}^{2}\right\rangle} \\
& =\sqrt{2 \pi} \frac{\left\langle B_{\mathrm{t}}^{2}\right\rangle}{\left\langle B_{0}^{2}\right\rangle}\left[\frac{\delta^{3}}{\left(\delta^{2}+2 W^{2}\right) \Delta^{\prime}}\right] e^{-\ell^{2} / 2\left(\delta^{2}+2 W^{2}\right)} .
\end{aligned}
$$

Finally, the broken curve shows what would be the expected contribution of the (assumed Gaussian) telescope beam alone to the width of the turbulent component (i.e., when $\delta=0$ in the argument of the exponential). Although we performed our analysis for a Gaussian turbulent correlation function, it is rather unlikely that the autocorrelation function of the turbulent magnetic field component fits this model. We therefore did not use the first few points (i.e., for $\ell \lesssim 0$.2) to fit Equation (53) to the data and concentrated on larger values of $\ell$ where it is more likely to obtain a reasonable fit. Indeed, there is evidence from the first few points, when $\ell \lesssim 0.2$, in the middle and bottom graphs of Figure 2 that the Gaussian turbulent autocorrelation function assumption is incorrect, as expected.

A comparison of the expected contribution of the telescope beam to the correlated turbulent component of the dispersion function (broken curve in the bottom graph of Figure 2) with the accompanying data reveals the imprint of the finite turbulent correlation length scale $\delta$ in the relative excess detected in the $0.1 \lesssim \ell \lesssim 0.4$ range. This imprint is also evident from the nonzero integrated ratio of the (square of the) turbulent to large-scale magnetic field strength ratio (i.e., $b^{2}(0)$ from Equation (55)) that is evident in the data through the intercept of the broken curves at $\ell=0$ in the top and middle graph of Figure 2. As is seen in Equation (55) this intercept will go to zero in the limit where $\delta \rightarrow 0$ when the number of turbulent cells subtended by the telescope beam $N$ tends to infinity (see Equation (52)), i.e., in cases where the turbulent component is basically completely integrated out.

The results from our fit of Equation (53) to the dispersion data are summarized in Table 1 . Most notably, we measure the turbulent correlation length to be $\delta \approx 16 \mathrm{mpc}$ (or $7^{\prime \prime} .3$ at $450 \mathrm{pc}$, the distance we adopt for OMC-1), which implies that there are on average $N \approx 21$ independent turbulent cells contained within the column of gas probed by our telescope beam. Furthermore, since our fit for the square of the "integrated" turbulent to largescale magnetic strength ratio yielded $b^{2}(0) \approx 0.013$, then it follows that

$$
\begin{aligned}
\frac{\left\langle B_{\mathrm{t}}^{2}\right\rangle}{\left\langle B_{0}^{2}\right\rangle} & =N b^{2}(0) \\
& \approx 0.28,
\end{aligned}
$$

where we used Equations (52) and (55). This quantity can be inserted in the Chandrasekhar-Fermi equation (Chandrasekhar \& Fermi 1953) to evaluate the strength plane-of-the-sky component of the large-scale magnetic field

$$
\begin{aligned}
\left\langle B_{0}^{2}\right\rangle^{1 / 2} & =\sqrt{4 \pi \rho} \sigma(v)\left[\frac{\left\langle B_{\mathrm{t}}^{2}\right\rangle}{\left\langle B_{0}^{2}\right\rangle}\right]^{-1 / 2} \\
& \approx 760 \mu \mathrm{G},
\end{aligned}
$$

where we used the same values as in Paper I for the mass density $\rho$ (i.e., a gas density of $10^{5} \mathrm{~cm}^{-3}$ and a mean molecular
Table 1

Results from Our Fit of Equation (53) to the Dispersion Data for OMC-1

\begin{tabular}{lcccccc}
\hline \hline & \multicolumn{3}{c}{ Fit Result } & & \multicolumn{3}{c}{ Derived Quantities } \\
\cline { 1 - 3 } \cline { 5 - 7 } $\begin{array}{l}\delta^{\mathrm{a}} \\
(\mathrm{mpc})\end{array}$ & $b^{2}(0)^{\mathrm{b}}$ & $\begin{array}{c}a_{2}^{\prime} \\
\left(\operatorname{arcmin}^{-2}\right)\end{array}$ & & $N^{\mathrm{c}}$ & $\left\langle B_{\mathrm{t}}^{2}\right\rangle /\left\langle B_{0}^{2}\right\rangle^{\mathrm{d}}$ & $\begin{array}{c}\left\langle B_{0}^{2}\right\rangle^{1 / 2} \mathrm{e} \\
(\mu \mathrm{G})\end{array}$ \\
\hline $16.0 \pm 0.4$ & $0.0134 \pm 0.001$ & $0.059 \pm 0.001$ & & 20.7 & $0.28 \pm 0.01$ & 760 \\
\hline
\end{tabular}

Notes.

${ }^{a}$ Corresponds to the fit result of 7.'3 30.2 at a distance of $450 \mathrm{pc}$ assumed for OMC-1.

${ }^{\mathrm{b}}$ Corresponds to the linear intercept of the broken curves at $\ell=0$ in the top and middle graphs of Figure 2 (see Equation (55)).

c Calculated using Equation (52) with the SHARP beam radius $W=4$ ".7 (or $\mathrm{FWHM}=11^{\prime \prime}$ ) and $\Delta^{\prime}=3 \cdot 5$.

${ }^{\mathrm{d}}$ Calculated by multiplying the fit result for $b^{2}(0)$ by $N$ (see Equations (52) and (55)).

e Calculated using the Chandrasekhar-Fermi equation (see Equation (57)) assuming a density of $10^{5} \mathrm{~cm}^{-3}$, a mean molecular weight of 2.3 , and a velocity dispersion of $1.85 \mathrm{~km} \mathrm{~s}^{-1}$. This estimate is probably not precise to better than a factor of a few due to the uncertainty in the density and $\Delta^{\prime}$.

weight of 2.3) and the one-dimensional velocity dispersion $\sigma(v)$ (i.e., $1.85 \mathrm{~km} \mathrm{~s}^{-1}$ as obtained from a representative $\mathrm{H}^{13} \mathrm{CO}^{+}$ $J=3 \rightarrow 2$ spectrum).

We are now in a better position to appreciate the importance of adequately taking into account the signal integration process. Indeed, failing to do so while using the ChandrasekharFermi equation would imply multiplying the value obtained in Equation (57) by $\sqrt{N} \approx 4$.6. This would yield a magnetic field strength of approximately $3.5 \mathrm{mG}$, a value that is certainly prohibitively high for OMC-1 (see Section 4.1).

\section{DISCUSSION}

\subsection{The Turbulent Power Spectrum}

The determination of the turbulent correlation $\delta$ length is important for the characterization of turbulence in molecular clouds. It is therefore desirable to compare our result of $\delta \approx$ $16 \mathrm{mpc}$ for OMC-1 with other independent techniques or analyses that seek to evaluate this quantity, or others related to it, either observationally or theoretically.

A parameter that is very closely related to $\delta$ is the ambipolar diffusion scale $\delta_{\mathrm{AD}}$ at which the ionized and neutral components of the gas decouple, and that may determine the cutoff wavelength of the power spectrum of the turbulent component of the magnetic field $\mathbf{B}_{\mathrm{t}}$. For this discussion, we therefore define $k_{\mathrm{AD}}=\delta_{\mathrm{AD}}^{-1}$ such that the (isotropic) turbulent power spectrum $\mathcal{R}_{\mathrm{t}}(k) \approx 0$ for $k>k_{\mathrm{AD}}$. It follows that if $\delta^{-1}$ is some measure of the width of $\mathcal{R}_{\mathrm{t}}(k)$ (e.g., its standard deviation), then we expect that $\delta \geqslant \delta_{\mathrm{AD}}$

Recent theoretical (Lazarian et al. 2004) and observational studies have yielded estimates of the order of $1 \mathrm{mpc}$ for $\delta_{\mathrm{AD}}$ in molecular clouds. Although an observational determination for the ambipolar diffusion scale has yet to be obtained for OMC1 , Li \& Houde (2008) have measured $\delta_{\mathrm{AD}} \approx 2 \mathrm{mpc}$ for the molecular cloud M17. If we assume for the moment that this value also applies to OMC-1, then we find that the turbulent correlation length scale of approximately $16 \mathrm{mpc}$ we measured, which is a factor of many larger than the quoted ambipolar diffusion cutoff scale, is consistent with the aforementioned expectation that $\delta \geqslant \delta_{\mathrm{AD}}$.

One should always keep in mind, however, that the value for $\delta_{\mathrm{AD}}$ determined by $\mathrm{Li} \&$ Houde (2008) pertains to a 
"Kolmogorov-like" turbulent power spectrum whereas our value for $\delta$ does not. That is, given a three-dimensional isotropic turbulent autocorrelation function ${ }^{7} \mathcal{R}_{\mathrm{t}}(\ell)$ from which one evaluates the correlation length $\delta$, the corresponding power spectrum $\mathcal{R}_{\mathrm{t}}(k)$ (of width $\delta^{-1}$ ) is specified by the Fourier transform of $\mathcal{R}_{\mathrm{t}}(\ell)$. The related Kolmogorov-like power spectrum is not $\mathcal{R}_{\mathrm{t}}(k)$, however, but is usually defined as $\mathcal{R}_{\mathrm{K}}(k) \equiv 4 \pi k^{2} \mathcal{R}_{\mathrm{t}}(k)$ (Frisch 1995). It follows that if $\delta_{\mathrm{AD}}$ is the ambipolar diffusion scale pertaining to the Kolmogorov-like spectrum $\mathcal{R}_{\mathrm{K}}(k)$, then we should define a turbulent correlation length $\delta_{\mathrm{K}}$ that also pertains to $\mathcal{R}_{\mathrm{K}}(k)$ for a meaningful comparison.

For example, if we consider the idealization of the Gaussian turbulent autocorrelation function of width $\delta$ used for our analysis

$$
\mathcal{R}_{\mathrm{t}}(\ell)=e^{-\ell^{2} / 2 \delta^{2}}
$$

then

$$
\mathcal{R}_{\mathrm{t}}(k)=(2 \pi)^{3 / 2} \delta^{3} e^{-\frac{1}{2} \delta^{2} k^{2}}
$$

and the Kolmogorov-like power spectrum is given by

$$
\mathcal{R}_{\mathrm{K}}(k)=2(2 \pi)^{5 / 2} \delta^{3} k^{2} e^{-\frac{1}{2} \delta^{2} k^{2}} .
$$

Calculating the (square of the) width $\delta_{\mathrm{K}}^{-1}$ of $\mathcal{R}_{\mathrm{K}}(k)$ (i.e., its variance) we have

$$
\begin{aligned}
\delta_{\mathrm{K}}^{-2} & =\frac{\int_{0}^{\infty} k^{2} \mathcal{R}_{\mathrm{K}}(k) d k}{\int_{0}^{\infty} \mathcal{R}_{\mathrm{K}}(k) d k} \\
& =3 \delta^{-2}
\end{aligned}
$$

Applying this relation to the result obtained for OMC-1, we now find that $\delta_{\mathrm{K}} \approx 9 \mathrm{mpc}$. This value is now a factor of almost 2 closer to theoretically and observationally expected values for $\delta_{\mathrm{AD}}$. Furthermore, we should also keep in mind that our assumption of Gaussian functions is probably incorrect. The true turbulent autocorrelation function could yield different values for $\delta$ and $\delta_{\mathrm{K}}$, which may be closer to the expected value for $\delta_{\mathrm{AD}}$.

Another fundamental parameter for the characterization of turbulence is the turbulent to large-scale magnetic field strength ratio. With the expectation that the magnetic field will be tied to the gas through flux freezing, which should apply for a significant part of the spectrum where $k<\delta_{\mathrm{AD}}^{-1}$, this parameter is a measure of the relative amount of turbulent energy contained in the gas. Our aforementioned determined value of 0.28 for the square of the turbulent to large-scale magnetic field strength ratio seems to indicate that turbulence does not dominate the dynamics in OMC-1.

As is shown in Equation (57), the square root of this ratio is used to calculate the strength of the plane-of-the-sky component of the large-scale magnetic field with

$$
\left[\frac{\left\langle B_{\mathrm{t}}^{2}\right\rangle}{\left\langle B_{0}^{2}\right\rangle}\right]^{1 / 2} \approx 0.53
$$

Although our estimate for the plane-of-the-sky component of the large-scale magnetic field of $\left\langle B_{0}^{2}\right\rangle^{1 / 2} \approx 760 \mu \mathrm{G}$ calculated using this ratio cannot be precise to better than a factor of a few because of uncertainties in the gas density and $\Delta^{\prime}$, this value is reasonable and in line with other independent measurements. For example, Crutcher et al. (1999) measured a line-of-sight

\footnotetext{
7 For this discussion, we do not restrict $\ell$ to the plane of the sky, but allow it to span the three dimensions.
}

magnetic field strength of $360 \mu \mathrm{G}$ in OMC-1 using CN Zeeman measurements, which probed densities that are comparable to those corresponding to our observations. Incidentally, using these values for the two components of the magnetic field we can get an estimate of approximately $65^{\circ}$ for the inclination angle of the large-scale magnetic field to the line of sight. Again, this value is consistent with the results obtained by Houde et al. (2004) for this object using an independent technique (i.e., $49^{\circ}$ in the Orion bar and $65^{\circ}$ at a location a few arcminutes northeast of Orion KL).

\subsection{Weaknesses of the Technique}

The use of the dispersion function in the polarization angle, while adequately taking into account the process of signal integration implicit to dust polarization measurements, allowed us to determine some of the fundamental parameters characterizing magnetized turbulence in molecular clouds. Our analysis rests, however, on a few assumptions that require some discussion:

1. Our definition for the integrated magnetic field $\overline{\mathbf{B}}(\mathbf{r})$ (see Equation (10)) does not perfectly mimic the measurement process through which dust polarization data are obtained. A consequence of this was noted in the discussion that followed Equation (31), where it was found that our analysis seemingly introduces a contribution to the angular dispersion that we do not expect to be present in actual data. Although a hypothetical analysis that would precisely duplicate the measurement process (see Equations (39)-(41)) would be desirable, the quest for an analytical solution renders this kind of idealization necessary. The same comment could be made for the assumptions of isotropy, homogeneity, and stationarity used throughout our calculations. Nonetheless, we expect that the results stemming from our treatment of the angular dispersion function are successful in the characterization of magnetized turbulence in molecular clouds.

2. We assumed a Gaussian form for the autocorrelation functions characterizing turbulence. This assumption is certainly incorrect and as a result it was not possible to obtain a reasonable fit when using part of the data where $\ell \lesssim 0.2$; the shortcomings of our Gaussian model are most clearly apparent in that region (see the discussion in Section 3.3). We must therefore keep in mind that our estimates for the turbulent correlation length $\delta$ and the turbulent to large-scale magnetic field strength ratio are correspondingly uncertain to some extent. On the other hand our fit is relatively robust, as small changes in the domain used for the fit (i.e., the range of values for $\ell$ ) do not lead to significantly different solutions. The same is true if a term proportional to $\ell^{4}$ is added to the large-scale function to be fitted (i.e., to the right-hand side of Equation (53)).

3. Another source of uncertainty is our modeling of the telescope beam using a Gaussian profile. Although the beam size we quote is based on actual measurements taken from chopped measurements on pointing sources (e.g., Uranus) and is consistent with other SHARP observations, we do not possess a detailed map of the telescope beam profile for our set of observations. The aforementioned uncertainty is a consequence of the fact that our determination of $\delta$ stems, in part, from a comparison of the correlated turbulent component of the dispersion function with the assumed Gaussian telescope beam (see the bottom graph of Figure 2). The significant difference observed between the 
two, however, makes it unlikely that the ensuing effect on our estimate of the turbulent length scale is important.

4. Our analysis has shown that the presence of turbulence in the polarized emission can bring about additional angular dispersion to that due to turbulence in the magnetic field. As was discussed in Section 3.1, however, our inability to disentangle these two contributions to the dispersion has forced us to introduce approximations that essentially brought about the neglect of the turbulent polarized emission. It should be noted that this implies that we overestimated the amount of turbulence in the magnetic field (measured through $\left\langle B_{\mathrm{t}}^{2}\right\rangle /\left\langle B_{0}^{2}\right\rangle$, as obtained through our fit to the data), which in turn translates into an underestimate of the largescale magnetic strength when using the ChandrasekharFermi equation. Although this effect is probably small, we once again stress that the effect of turbulence in the polarized emission could perhaps be advantageously investigated and quantified through simulations.

It is interesting to note that although we limited ourselves to the determination of only two parameters characterizing the turbulent power spectrum, our technique can in principle be used to achieve significantly more. We can verify this statement by considering the Fourier transform $b^{2}\left(k_{v}\right)$ associated with the turbulent component (see Equation (55), for example), which can be determined from Equation (A12)

$$
b^{2}\left(k_{v}\right)=\frac{1}{\left\langle B_{0}^{2}\right\rangle}\left\|H\left(\mathbf{k}_{v}\right)\right\|^{2}\left[\int \mathcal{R}_{\mathrm{t}}\left(\mathbf{k}_{v}, k_{u}\right) \operatorname{sinc}^{2}\left(\frac{k_{u} \Delta}{2}\right) d k_{u}\right],
$$

where $\mathcal{R}_{\mathrm{t}}\left(\mathbf{k}_{v}, k_{u}\right)$ is the Fourier transform of $\mathcal{R}_{\mathrm{t}}(v, u) \equiv$ $R_{F, \mathrm{t}}(v, u) R_{\mathbf{B}, \mathrm{t}}(v, u)$. Since the spatial frequency component $k_{u}$ of $\mathcal{R}_{\mathrm{t}}\left(\mathbf{k}_{v}, k_{u}\right)$ is eliminated through the corresponding integration, we can equally write

$$
b^{2}\left(k_{v}\right)=\frac{1}{\left\langle B_{0}^{2}\right\rangle}\left\|H\left(\mathbf{k}_{v}\right)\right\|^{2} \mathcal{R}_{t}\left(\mathbf{k}_{v}\right)
$$

where $\mathcal{R}_{\mathrm{t}}\left(\mathbf{k}_{v}\right)$ is now interpreted as the two-dimensional turbulent power spectrum. Under the assumption of isotropy it would be expected that $\mathcal{R}_{\mathrm{t}}\left(\mathbf{k}_{v}\right)$ is similar in form to $\mathcal{R}_{\mathrm{t}}(k)$, where $\mathcal{R}_{\mathrm{t}}(k)$ is the three-dimensional turbulent power spectrum defined in Section 4.1. Since the left-hand side of Equation (64) can be evaluated by taking a Fourier transform of the data (as shown with the symbols in the bottom graph of Figure 2) and that the beam profile $H\left(\mathbf{k}_{v}\right)$ is presumably well characterized, it follows that the turbulent power spectrum $\mathcal{R}_{\mathrm{t}}\left(\mathbf{k}_{v}\right)$ can readily be determined through the inversion of Equation (64) (using a simple Wiener optimal filter, for example).

Unfortunately our polarization map does not have enough spatial resolution to allow us to perform this analysis (i.e., our beam profile $H\left(\mathbf{k}_{v}\right)$ is much too narrow in frequency space), but there is no obvious reason why this should not be feasible with higher resolution observations. A much better characterization of the turbulent power spectrum would then result. We will address this question in a subsequent publication.

\section{SUMMARY}

In this paper, we expanded our study on the dispersion of polarization angles in molecular clouds and showed how the effect of signal integration through the thickness of the cloud as well as across the area subtended by the telescope beam inherent to dust continuum measurements can be incorporated in our analysis. We correctly accounted for its effect on the measured angular dispersion and inferred turbulent to largescale magnetic field strength ratio. We also showed how to evaluate the turbulent magnetic field correlation scale from polarization data and applied our results to the molecular cloud $\mathrm{OMC}-1$. For this object, we find a turbulent correlation length of $\delta \approx 16 \mathrm{mpc}$, a turbulent to large-scale magnetic field strength ratio of approximately 0.5 , and a plane-of-the-sky large-scale magnetic field strength $\left\langle B_{0}^{2}\right\rangle^{-1 / 2} \approx 760 \mu \mathrm{G}$.

In future papers, we will extend our technique to study the possibility for obtaining a detailed characterization of the turbulent power spectrum, as well as the discussing evidence for anisotropy in magnetized turbulence from dust polarization data.

M.H.'s research is funded through the NSERC Discovery Grant, Canada Research Chair, Canada Foundation for Innovation, Ontario Innovation Trust, and Western's Academic Development Fund programs. J.E.V. acknowledges support from the CSO, which is funded through NSF AST 08-38261 and AST 05-40882. This work has also been supported in part by NSF grants AST 05-05230, AST 02-41356, and AST 05-05124.

\section{APPENDIX A \\ DERIVATIONS}

\section{A.1. The Autocorrelation Function of the Integrated Magnetic Field}

To obtain Equation (11) we start with

$$
\langle\overline{\mathbf{B}}(\mathbf{r}) \cdot \overline{\mathbf{B}}(\mathbf{r}+\boldsymbol{\ell})\rangle=\iiint \int H(\mathbf{r}-\mathbf{a}) H\left(\mathbf{r}+\boldsymbol{\ell}-\mathbf{a}^{\prime}\right)\left[\frac{1}{\Delta^{2}} \int_{0}^{\Delta} \int_{0}^{\Delta} R_{F}(v, u) R_{\mathbf{B}}(v, u) d z^{\prime} d z\right] d^{2} a^{\prime} d^{2} a
$$

from Equations (10), (12), (13), $u=\left|z^{\prime}-z\right|$, and $v=\left|\mathbf{a}^{\prime}-\mathbf{a}\right|$. The integral

$$
I(v)=\frac{1}{\Delta^{2}} \int_{0}^{\Delta} \int_{0}^{\Delta} R_{F}(v, u) R_{\mathbf{B}}(v, u) d z^{\prime} d z
$$

can be transformed into a one-dimensional integral on account of the assumed stationarity of the functions present in the integrand (i.e., they are solely a function of $\left|z^{\prime}-z\right|$, besides $v$ ). To accomplish this, after making the change of variable $u=\left|z^{\prime}-z\right|$, we integrate over the square surface delimited by $0 \leqslant z \leqslant \Delta$ and $0 \leqslant z^{\prime} \leqslant \Delta$ along the linear path where $-\Delta \leqslant u \leqslant \Delta$. This path is also perpendicular to a family of strips (along which $u$ is constant) of infinitesimal area

$$
d S=\Delta\left(1-\frac{|u|}{\Delta}\right) d u
$$


to first order in $d u$. We then find that

$$
I(v)=\frac{2}{\Delta} \int_{0}^{\Delta}\left(1-\frac{u}{\Delta}\right) R_{F}(v, u) R_{\mathbf{B}}(v, u) d u .
$$

We can now further make the change of variables $\mathbf{a} \rightarrow \mathbf{r}-\mathbf{a}$ and $\mathbf{a}^{\prime} \rightarrow \mathbf{r}-\mathbf{a}^{\prime}$, which is then inserted with Equation (A2) into Equation (A1) to yield

$$
\langle\overline{\mathbf{B}} \cdot \overline{\mathbf{B}}(\ell)\rangle=\iiint \int H(\mathbf{a}) H\left(\mathbf{a}^{\prime}+\ell\right)\left[\frac{2}{\Delta} \int_{0}^{\Delta}\left(1-\frac{u}{\Delta}\right) R_{F}(v, u) R_{\mathbf{B}}(v, u) d u\right] d^{2} a^{\prime} d^{2} a,
$$

where any dependence on $\mathbf{r}$ is done away with since $u$ and $v$ are unaffected by this change of variables and we integrate over all of space.

Equation (24) can be derived by first noting that

$$
\frac{2}{\Delta} \int_{0}^{\Delta}\left(1-\frac{u}{\Delta}\right) e^{-\left(v^{2}+u^{2}\right) / 2 \delta^{2}} d u \simeq \sqrt{2 \pi}\left(\frac{\delta}{\Delta}\right) e^{-v^{2} / 2 \delta^{2}}
$$

when $\delta \ll \Delta$. This result will arise for all terms containing a turbulent component (i.e., $\left\langle B_{\mathrm{t}}^{2}\right\rangle$ and/or $\left.\left\langle F_{\mathrm{t}}^{2}\right\rangle\right)$ in Equation (A5).

Setting $\mathbf{u}=\mathbf{a}^{\prime}+\mathbf{a}$ and $\mathbf{v}=\mathbf{a}^{\prime}-\mathbf{a}$ we have

$$
\begin{aligned}
\frac{1}{2 W^{2}}\left(|\mathbf{a}|^{2}+\left|\mathbf{a}^{\prime}+\ell\right|^{2}\right)+\frac{1}{2 \delta^{2}}\left|\mathbf{a}^{\prime}-\mathbf{a}\right|^{2} & =\frac{1}{4 W^{2}}\left(|\mathbf{u}+\ell|^{2}+|\mathbf{v}+\ell|^{2}\right)+\frac{|\mathbf{v}|^{2}}{2 \delta^{2}} \\
& =\frac{|\mathbf{u}+\ell|^{2}}{4 W^{2}}+\left(\frac{\delta^{2}+2 W^{2}}{4 \delta^{2} W^{2}}\right)\left|\mathbf{v}+\ell\left(\frac{\delta^{2}}{\delta^{2}+2 W^{2}}\right)\right|^{2}+\frac{\ell^{2}}{2\left(\delta^{2}+2 W^{2}\right)}
\end{aligned}
$$

It follows from this relation, the Jacobian related to the coordinate transformation above, and Equation (23) that

$$
\iiint \int H(\mathbf{a}) H\left(\mathbf{a}^{\prime}+\ell\right) e^{-\left|\mathbf{a}^{\prime}-\mathbf{a}\right|^{2} / 2 \delta^{2}} d^{2} a^{\prime} d^{2} a=\frac{e^{-\ell^{2} / 2\left(\delta^{2}+2 W^{2}\right)}}{4\left(2 \pi W^{2}\right)^{2}}\left[\int e^{-u^{2} / 4 W^{2}} d u \cdot \int e^{-\left(\frac{\delta^{2}+2 W^{2}}{4 \delta^{2} W^{2}}\right) v^{2}} d v\right]^{2}=\left(\frac{\delta^{2}}{\delta^{2}+2 W^{2}}\right) e^{-\ell^{2} / 2\left(\delta^{2}+2 W^{2}\right)},
$$

which is dependent on only $\ell$ and not its orientation. Equation (24) follows from Equations (11), (21), (22), (25)-(28), (A6), and (A8).

\section{A.2. The Length Scale of the Large-scale Component}

We have used the region where the distance $\ell$ is less than a few times the beam radius to fit the dispersion data to Equation (43). It is therefore appropriate to inquire as to the validity of this procedure. To do so, we first note that the autocorrelation function given by Equation (11) can be expanded with its Fourier transform as follows (with $\mathcal{R}(v, u) \equiv R_{F}(v, u) R_{\mathbf{B}}(v, u)$ ):

$$
\begin{aligned}
\langle\overline{\mathbf{B}}(\mathbf{r}) \cdot \overline{\mathbf{B}}(\mathbf{r}+\ell)\rangle & =\iiint \int H(\mathbf{a}) H\left(\mathbf{a}^{\prime}+\ell\right)\left[\frac{1}{\Delta} \int_{-\Delta}^{\Delta}\left(1-\frac{u}{\Delta}\right) \mathcal{R}(v, u) d u\right] d^{2} a^{\prime} d^{2} a \\
& =\iiint \int H(\mathbf{a}) H\left(\mathbf{a}^{\prime}+\ell\right)\left\{\frac{1}{\Delta} \int_{-\Delta}^{\Delta}\left(1-\frac{|u|}{\Delta}\right)\left[\frac{1}{(2 \pi)^{3}} \iiint \mathcal{R}\left(\mathbf{k}_{v}, k_{u}\right) e^{i \mathbf{k} \cdot \mathbf{x}} d^{3} k\right] d u\right\} d^{2} a^{\prime} d^{2} a \\
& =\frac{1}{(2 \pi)^{3}} \iiint \mathcal{R}\left(\mathbf{k}_{v}, k_{u}\right)\left[\iiint \int H(\mathbf{a}) H\left(\mathbf{a}^{\prime}+\ell\right) e^{i \mathbf{k}_{v} \cdot\left(\mathbf{a}-\mathbf{a}^{\prime}\right)} d^{2} a^{\prime} d^{2} a\right]\left[\frac{1}{\Delta} \int_{-\Delta}^{\Delta}\left(1-\frac{|u|}{\Delta}\right) e^{i k_{u} u} d u\right] d^{3} k \\
& =\frac{1}{(2 \pi)^{3}} \iiint \mathcal{R}\left(\mathbf{k}_{v}, k_{u}\right)\left[H\left(\mathbf{k}_{v}\right) H\left(-\mathbf{k}_{v}\right) e^{i \mathbf{k}_{v} \cdot \ell}\right]\left[\frac{1}{\Delta} \int_{-\Delta}^{\Delta}\left(1-\frac{|u|}{\Delta}\right) e^{i k_{u} u} d u\right] d^{3} k,
\end{aligned}
$$

where the Fourier transform of a function is represented by simply replacing the spatial arguments by their k-space counterparts (e.g., $\left.\mathcal{R}(v, u) \rightleftharpoons \mathcal{R}\left(\mathbf{k}_{v}, k_{u}\right)\right)$, and $\mathbf{x}=\mathbf{v}+u \mathbf{e}_{z}$ with $\mathbf{v}=\mathbf{a}^{\prime}-\mathbf{a}$ and $u=\left|z^{\prime}-z\right|$. But since

$$
\begin{aligned}
\frac{1}{\Delta} \int_{-\Delta}^{\Delta}\left(1-\frac{|u|}{\Delta}\right) e^{i k_{u} u} d u & =\frac{1}{\Delta^{2}} \int\left[\int \operatorname{rect}\left(\frac{\tau}{\Delta}\right) \operatorname{rect}\left(\frac{u-\tau}{\Delta}\right) d \tau\right] e^{i k_{u} u} d u \\
& =\operatorname{sinc}^{2}\left(\frac{k_{u} \Delta}{2}\right),
\end{aligned}
$$

with

$$
\operatorname{rect}\left(\frac{\tau}{\Delta}\right)= \begin{cases}1, & |\tau|<\frac{\Delta}{2} \\ 0, & |\tau|>\frac{\Delta}{2}\end{cases}
$$


we can write

$$
\langle\overline{\mathbf{B}}(\mathbf{r}) \cdot \overline{\mathbf{B}}(\mathbf{r}+\ell)\rangle=\frac{1}{(2 \pi)^{2}} \iint\left\|H\left(\mathbf{k}_{v}\right)\right\|^{2}\left[\frac{1}{2 \pi} \int \mathcal{R}\left(\mathbf{k}_{v}, k_{u}\right) \operatorname{sinc}^{2}\left(\frac{k_{u} \Delta}{2}\right) d k_{u}\right] e^{i \mathbf{k}_{v} \cdot \ell} d^{2} k_{v},
$$

where $\left\|H\left(\mathbf{k}_{v}\right)\right\|^{2}=H\left(\mathbf{k}_{v}\right) H^{*}\left(\mathbf{k}_{v}\right)$ and $\operatorname{sinc}(x) \equiv \sin (x) / x$.

The effect of signal integration is made explicit in Equation (A12). More precisely, it is seen that the integration through the thickness of the cloud heavily filters the spectral components along the line of sight to a small set symmetrically located about $k_{u}=0$ through the presence of $\operatorname{sinc}^{2}\left(k_{u} \Delta\right)$ and the integration along $k_{u}$, while the integration across the beam profile on the plane of the sky further filters the signal with $\left\|H\left(\mathbf{k}_{v}\right)\right\|^{2}$. For a Gaussian profile as specified by Equation (23) we have

$$
H\left(\mathbf{k}_{v}\right)=e^{-\frac{1}{2} W^{2} k_{v}^{2}},
$$

and it becomes clear to the only spectral components that remain in the integrated polarization map satisfy $k_{v} \lesssim W^{-1}$; the larger the telescope beam the more heavily the signal is filtered spatially.

It should also be noted that the derivation of Equation (24), which was demonstrated in Section A.1, can also be achieved using Equation (A12) under the assumption of Gaussian turbulent autocorrelation functions (see Equations (21) and (22)) with $\delta \ll \Delta$.

Restricting Equation (A12) to its large-scale component, assuming isotropy, and referring to Equation (28) we can write

$$
\begin{aligned}
\langle\alpha(\ell)\rangle & =\frac{1}{(2 \pi)^{2}} \int_{0}^{2 \pi} \int_{0}^{\infty}\left\|H\left(k_{v}\right)\right\|^{2}\left[\frac{1}{2 \pi} \int \frac{\mathcal{R}_{0}\left(k_{v}, k_{u}\right)}{\left\langle F_{0}^{2}\right\rangle\left\langle B_{0}^{2}\right\rangle} \operatorname{sinc}^{2}\left(\frac{k_{u} \Delta}{2}\right) d k_{u}\right] e^{i k_{v} \ell \cos (\phi)} k_{v} d k_{v} d \phi \\
& =\frac{1}{2 \pi} \int_{0}^{\infty}\left\|H\left(k_{v}\right)\right\|^{2} J_{0}\left(k_{v} \ell\right)\left[\frac{1}{2 \pi} \int \frac{\mathcal{R}_{0}\left(k_{v}, k_{u}\right)}{\left\langle F_{0}^{2}\right\rangle\left\langle B_{0}^{2}\right\rangle} \operatorname{sinc}^{2}\left(\frac{k_{u} \Delta}{2}\right) d k_{u}\right] k_{v} d k_{v} .
\end{aligned}
$$

where $\mathcal{R}_{0}(v, u)=R_{F, 0}(v, u) R_{\mathbf{B}, 0}(v, u)$ and $J_{0}(x)$ is the Bessel function of the first kind of order 0. Performing a Taylor expansion about $\ell=0$ using the identity

$$
J_{0}(x)=\sum_{n=0}^{\infty}(-1)^{n} \frac{\left(x^{2} / 4\right)^{n}}{(n !)^{2}}
$$

it is clear that, as expected, a Taylor expansion of $\langle\alpha(\ell)\rangle$ only contains terms of even power. Equations (A13) and (A14) show that, once again, the beam filtering process will remove any spectral component with $k_{v} \gtrsim W^{-1}$ in the large-scale dispersion function; its characteristic length scale is therefore constrained by, and limited to a few times, the telescope beam radius. Because of this beam filtering effect we expect the part of the spectrum at $k_{v} \lesssim W^{-1}$ to be dominant in Equation (A14), therefore limiting ourselves to a domain where $\ell$ is less than a few times the beam radius (i.e., $k_{v} \ell \lesssim 1$ ) to fit the dispersion data with Equation (43) implies that

$$
J_{0}\left(k_{v} \ell\right) \simeq 1-\frac{\left(k_{v} \ell\right)^{2}}{4}
$$

which justifies the method used to model that data and extract the needed information from it.

\section{APPENDIX B}

\section{DATA ANALYSIS}

The data for OMC-1 from the SHARP polarimeter studied here have been previously published by Vaillancourt et al. (2008). For our purposes we only include data that satisfy the $p>3 \sigma_{p}$ criterion, where $p$ is the polarization fraction and $\sigma_{p}$ its uncertainty.

The angle differences between each and every pair of data points are calculated as

$$
\Delta \Phi_{i j}=\Phi_{i}-\Phi_{j}
$$

and the corresponding distance between each point

$$
\ell_{i j} \equiv\left|\mathbf{r}_{i}-\mathbf{r}_{j}\right|
$$

Note that $\ell_{i j}=\ell_{j i}$ so that a map with $N$ data points contains only $N(N-1) / 2$ distinct differences. Also note that $\left|\Delta \Phi_{i j}\right|$ is constrained to be in the range $[0,90] \mathrm{deg}$.

These data are divided into separate distance bins with sizes corresponding to integer multiples of the grid spacing $\Delta \ell$ that results after processing the SHARP map $(\Delta \ell=2$ '.37; note that a SHARP pixel is approximately 4". 6 and the beam FWHM for our polarization map is approximately $11^{\prime \prime}$ ); the bin for $\ell_{k}$ (which corresponds to $k$ pixels) covers $\left(\ell_{k}-\Delta \ell / 2\right) \leqslant \ell_{i j}<\left(\ell_{k}+\Delta \ell / 2\right)$. Within each bin $k$ we thus calculate the dispersion function with

$$
1-\left\langle\cos \left(\Delta \Phi_{i j}\right)\right\rangle_{k}, \text { for all }\left(\ell_{k}-\Delta \ell / 2\right) \leqslant \ell_{i j}<\left(\ell_{k}+\Delta \ell / 2\right) .
$$

The dispersion function is corrected for measurement uncertainty within each bin according to the uncertainty on each $\Delta \Phi_{i j}$ and propagating the measurement uncertainties on both $\Phi_{i}$ and $\Phi_{j}$ available in the data set, as is explained below. However, since it is 
often the case that $\ell_{i j}<W$ for some values of $\Delta \Phi_{i j}$ and their corresponding uncertainty will be correlated. We have therefore used the following relation for the measurement uncertainty:

$$
\sigma^{2}\left(\Delta \Phi_{i j}\right) \simeq \sigma^{2}\left(\Phi_{i}\right)+\sigma^{2}\left(\Phi_{j}\right)-2 \sigma\left(\Phi_{i}\right) \sigma\left(\Phi_{j}\right) e^{-\ell_{i j}^{2} / 4 W^{2}},
$$

which exhibits the right behavior when $\ell_{i j}=0$ and $\ell_{i j} \gtrsim 2 W$. The data are then corrected for measurement uncertainty with

$$
\begin{aligned}
\left\langle\cos \left(\Delta \Phi_{i j}\right)\right\rangle_{k, 0} & =\frac{\left\langle\cos \left(\Delta \Phi_{i j}\right)\right\rangle_{k}}{\left\langle\cos \left[\sigma\left(\Delta \Phi_{i j}\right)\right]\right\rangle_{k}} \\
& \simeq \frac{\left\langle\cos \left(\Delta \Phi_{i j}\right)\right\rangle_{k}}{1-\frac{1}{2}\left\langle\sigma^{2}\left(\Delta \Phi_{i j}\right)\right\rangle_{k}},
\end{aligned}
$$

where $\left\langle\cos \left(\Delta \Phi_{i j}\right)\right\rangle_{k, 0}$ is the quantity used in our analysis and for the plots in Figure 2, and an even probability distribution was assumed for the measurement uncertainty in $\Delta \Phi_{i j}$.

Finally, the measurement uncertainties for the dispersion function $1-\left\langle\cos \left(\Delta \Phi_{i j}\right)\right\rangle_{k, 0}$ is determined with

$$
\sigma^{2}\left[\left\langle\cos \left(\Delta \Phi_{i j}\right)\right\rangle_{k, 0}\right]=\left\langle\sin \left(\Delta \Phi_{i j}\right)\right\rangle_{k}^{2}\left\langle\sigma^{2}\left(\Delta \Phi_{i j}\right)\right\rangle_{k}+\frac{3}{4}\left\langle\cos \left(\Delta \Phi_{i j}\right)\right\rangle_{k}^{2}\left\langle\sigma^{4}\left(\Delta \Phi_{i j}\right)\right\rangle_{k},
$$

for all $\left(\ell_{k}-\Delta \ell / 2\right) \leqslant \ell_{i j}<\left(\ell_{k}+\Delta \ell / 2\right)$. These uncertainties are those plotted in Figure 2, most of them are too small to be seen in the figure, especially at the smallest displacements.

\section{APPENDIX C}

\section{LIST OF SYMBOLS}

In this appendix we list and define symbols for important variables and functions appearing in the text, as well as given in parentheses the number of the equation where they are first introduced or defined.

1. b: normalized magnetic field (Equation (3));

2. $\overline{\mathbf{b}}$ : integrated normalized magnetic field (Equation (4));

3. $b^{2}(\ell)$ : correlated turbulent component (Equation (55));

4. B: total magnetic field (Equation (2));

5. $\mathbf{B}_{0}$ : large-scale magnetic field (Equation (2));

6. $\mathbf{B}_{\mathrm{t}}$ : turbulent magnetic field (Equation (2));

7. $\overline{\mathbf{B}}$ : integrated total magnetic field (Equation (10));

8. $\overline{\mathbf{B}}_{0}$ : integrated large-scale magnetic field (Equation (10));

9. $\overline{\mathbf{B}}_{\mathrm{t}}$ : integrated turbulent magnetic field (Equation (10));

10. $\mathbf{e}_{r}$ : unit vector along $\mathbf{r}$ on the plane-of-the-sky (Equation (5));

11. $\mathbf{e}_{z}$ : unit vector along the line of sight within a molecular cloud (Equation (5));

12. $F$ : total polarized emission (Equation (4));

13. $F_{0}$ : large-scale polarized emission (Equation (15));

14. $F_{\mathrm{t}}$ : turbulent polarized emission (Equation (15));

15. $\bar{F}$ : integrated total polarized emission (Equation (38));

16. $H$ : telescope beam profile (Equation (4));

17. $J_{0}$ : Bessel function of the first kind of order 0 (Equation (A15));

18. $\ell$ : distance between two measurement positions (Equation (1));

19. $N$ : number of independent turbulent cells in the gas column probed by the beam (Equations (29) and (52));

20. $P$ : polarized flux (Equation (41));

21. $\bar{P}$ : integrated polarized flux (Equation (41));

22. $Q$ : Stokes parameter (Equation (39));

23. $\bar{Q}$ : integrated Stokes parameter (Equation (39));

24. r: position vector on the plane-of-the-sky (Equation (5));

25. $R_{\mathbf{B}}$ : autocorrelation function of the total magnetic field (Equation (12));

26. $R_{\mathbf{B}, 0}$ : autocorrelation function of the large-scale magnetic field (Equation (18));

27. $R_{\mathbf{B}, t}$ : autocorrelation function of the turbulent magnetic field (Equation (18));

28. $R_{F}$ : autocorrelation function of the total polarized emission (Equation (13));

29. $R_{F, 0}$ : autocorrelation function of the large-scale polarized emission (Equation (19));

30. $R_{F, \mathrm{t}}$ : autocorrelation function of the turbulent polarized emission (Equation (19));

31. $\mathcal{R}$ : product of $R_{F}$ and $R_{\mathbf{B}}$ (in normal space; Equation (A9));

32. $\mathcal{R}_{0}$ : product of $R_{F, 0}$ and $R_{\mathbf{B}, 0}$ (in normal space; Equation (46)); 
33. $\mathcal{R}_{\mathrm{K}}(k)$ : Kolmogorov-like power spectrum (Equation (60));

34. $\mathcal{R}_{\mathrm{t}}$ : turbulent correlation function (Equation (58));

35. $U$ : Stokes parameter (Equation (40));

36. $\bar{U}$ : integrated Stokes parameter (Equation (40));

37. $W$ : telescope beam radius (Equation (23));

38. $\mathbf{x}$ : three-dimensional position vector (Equation (5));

39. $z$ : position along the line of sight within a molecular cloud (Equation (5));

40. $\alpha(\ell)$ : normalized large-scale function (Equation (28));

41. $\delta$ : turbulent correlation length scale of the magnetic field (Equation (21));

42. $\delta^{\prime}$ : turbulent correlation length scale of the polarized emission (Equation (22));

43. $\delta_{\mathrm{K}}^{-1}$ : Kolomogorov spectral width (Equation (61));

44. $\Delta$ : maximum depth of a molecular cloud along the line of sight (Equation (4));

45. $\Delta^{\prime}$ : effective depth of the molecular cloud along the line of sight (Equation (34));

46. $\Delta k_{u}$ : spectral width of $\mathcal{R}_{0}$ (Equation (47));

47. $\Delta \Phi(\ell)$ : difference in polarization angles between two positions separated by $\ell$ (Equation (1));

48. $\langle\cdots\rangle$ : average of some quantity (Equation (1)).

\section{REFERENCES}

Chandrasekhar, S., \& Fermi, E. 1953, ApJ, 118, 113

Crutcher, R. M., Troland, T. H., Lazareff, B., Paubert, G., \& Kazés, I. 1999, ApJ, 514, L121

Dotson, J. L., Davidson, J. A., Dowell, C. D., Hildebrand, R. H., Kirby, L., \& Vaillancourt, J. E. 2009, ApJS, submitted

Falceta-Gonçalves, D., Lazarian, A., \& Kowal, G. 2008, ApJ, 679, 537

Frisch, U. 1995, Turbulence: The Legacy of A. N. Kolmogorov (Cambridge: Cambridge Univ. Press)

Heitsch, F., Zweibel, E. G., Mac Low, M.-M., Li, P., \& Norman, M. L. 2001, ApJ, 561,800

Hildebrand, R. H., Kirby, L., Dotson, J. L., Houde, M., \& Vaillancourt, J. E. 2009, ApJ, 696, 567 (Paper I)

Houde, M., Dowell, C. D., Hildebrand, R. H., Dotson, J. L., Vaillancourt, J. E., Phillips, T. G., Peng, R., \& Bastien, P. 2004, ApJ, 604, 717
Kudoh, T., \& Basu, S. 2003, ApJ, 595, 842

Lazarian, A., Vishniac, E. T., \& Cho, J. 2004, ApJ, 603, 180

Li, H., Attard, M., Dowell, C. D., Hildebrand, R. H., Houde, M., Kirby, L. Novak, G., \& Vaillancourt, J. E. 2006, Proc. SPIE, 6275, 48

Li, H., Dowell, C. D., Kirby, L., Novak, G., \& Vaillancourt, J. E. 2008, Appl. Opt., 47, 422

Li, H., \& Houde, M. 2008, ApJ, 677, 1151

Matthews, B. C., McPhee, C., Fissel, L., \& Curran, R. L. 2009, ApJS, 182, 143

Myers, P. C., \& Goodman, A. A. 1991, ApJ, 373, 509

Novak, G., et al. 2004, Proc. SPIE, 5498, 278

Ostriker, E. C., Stone, J. M., \& Gammie, C. F. 2001, ApJ, 546, 980

Padoan, P., Goodman, A. A., Draine, B. T., Juvela, M., Nordland, Å., \& Rögnvaldsson, Ö. E. 2001, ApJ, 559, 1005

Vaillancourt, J. E., et al. 2008, ApJ, 675, L25

Wiebe, D. S., \& Watson, W. D. 2004, ApJ, 615, 314 